\title{
Otitis media aguda: generalidades y resistencia antibiótica
}

Acute otitis media: generalities and antibiotic resistance

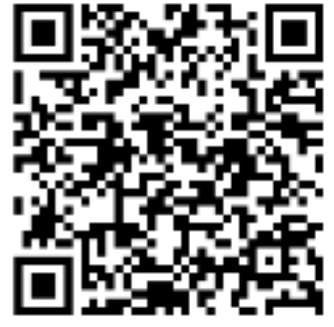

${ }^{1}$ Médico general, graduada de la Universidad de Costa Rica (UCR). Código médico: 15154 squesadasolano@gmail.com

${ }^{2}$ Médico general, graduado de la Universidad de Costa Rica (UCR). Codigo médico: 15165 fidel1mackenzie@gmail.com

${ }^{3}$ Médico general, graduado de la Universidad de Costa Rica (UCR). Código médico: 16337 melvincortes812@gmail.com

\author{
${ }^{1}$ Dra. Stephannie Quesada Solano \\ Hospital San Carlos, Alajuela, Costa Rica \\ squesadasolano@gmail.com \\ https://orcid.org/0000-0002-2593-338X
}

${ }^{2}$ Dr. Fidel Mackenzie Visbal Hospital San Francisco de Asís, Alajuela, Costa Rica fidel1mackenzie@gmail.com

https://orcid.org/0000-0002-4752-3496

${ }^{3}$ Dr. Melvin Cortés Mejía Investigador independiente, San José, Costa Rica melvincortes812@gmail.com

https://orcid.org/0000-0002-2310-3139

RECIBIDO

CORREGIDO

ACEPTADO

$28 / 03 / 2019$

$06 / 04 / 2019$

$15 / 04 / 2019$

\section{RESUMEN}

La otitis media $(\mathrm{OM})$ es uno de los diagnósticos más comunes en pediatría y constituye la indicación más frecuente de prescripción de antibióticos en la infancia. Para el diagnóstico de la otitis media aguda (OMA) se tienen en cuenta hallazgos de la otoscopia, diversos signos clínicos y el tiempo de evolución de los síntomas principales. La decisión de iniciar antibioticoterapia debe fundamentarse en la estratificación de riesgo del paciente. La espera vigilante como alternativa a la antibioticoterapia inmediata en casos seleccionados reduce el uso de antibióticos sin aumentar el riesgo de daño al paciente y ha demostrado ser una medida costo-efectiva. El fármaco de elección para el tratamiento empírico de la OMA es la amoxicilina. Otras alternativas frecuentemente utilizadas son las cefalosporinas, macrólidos o combinaciones con inhibidores de $\beta$-lactamasas. El Streptococcus pneumoniae resistente a la penicilina se considera la causa principal de fracaso terapéutico de los antibióticos en la OMA. Con la introducción de la vacuna antineumocócica conjugada heptavalente se han observado cambios en la frecuencia de determinados serotipos de Streptococcus pneumoniae y un aumento en el aislamiento de Haemophilus influenzae en las muestras de fluido del oído medio de paciente con OMA. Estudios recientes han 
evidenciado el aumento en la prevalencia de agentes productores de $\beta$-lactamasas en pacientes con OMA.

PALABRAS CLAVE: Otitis media; espera vigilante; Streptococcus pneumoniae; amoxicilina; farmacorresistencia microbiana.

\section{ABSTRACT}

Otitis media $(\mathrm{OM})$ is one of the most common diagnoses in pediatric population and the most frequent indication for the prescription of antibiotics in childhood. The diagnosis of acute otitis media (AOM) is based on the findings of the otoscopy, various clinical signs and the time of evolution of the main symptoms. The decision to initiate antibiotic therapy should be based on the risk stratification of the patient. An alternative observation and close follow-up strategy in selected cases help to reduce the antibiotic exposure, and has also proven to be a cost-effective measure. The drug of choice for the empirical treatment of AOM is amoxicillin. Other treatment options are cephalosporins, macrolides or combinations with $\beta$-lactamase inhibitors. Streptococcus pneumoniae resistant to penicillin is the main cause of the therapeutic failure of antibiotics in AOM. The introduction of the pneumococcal conjugate vaccine has changed the frequency of the serotypes of Streptococcus pneumoniae, and has increased the isolation of Haemophilus influenzae in the fluid samples of the middle ear of the patients with AOM. Recent studies have shown an increase in the prevalence of $\beta$-lactamase producer agents in patients with AOM.

KEYWORDS: Otitis media; watchful waiting; Streptococcus pneumoniae; amoxicillin; drug resistance, microbial.

\section{INTRODUCCIÓN}

La otitis media (OM) es uno de los diagnósticos más comunes en pediatría y constituye la indicación más frecuente de prescripción de antibióticos en la infancia $(1,2)$. El término OM hace referencia a la inflamación del oído medio, que se caracteriza por la presencia de efusión y ocasiona otalgia y pérdida auditiva (1). Además, en la OMA hay signos y síntomas de infección aguda, como fiebre $(1,3)$.

La OMA tiene su máxima incidencia entre los 6 y 12 meses de edad, y se estima que el $90 \%$ de los niños entre los 3 meses y 2 años de edad han presentado al menos un episodio de OMA (4).

La mayor parte de los casos de OMA resuelve de forma espontánea; no obstante, el $30-40 \%$ de los niños entre 2 y 5 años presentan cuadros recurrentes y más del $10 \%$ no mejora a pesar de administrar tratamiento antibiótico $(4,5)$. Dentro de los factores de riesgo conocidos para OMA recurrente, se encuentran episodios de OMA previos, ciclos previos de antibioticoterapia y edad menor a 2 años (5).

Algunas complicaciones serias de la OMA son la otitis media supurativa crónica, que se acompaña de descarga 
persistente a través de una perforación timpánica, y la mastoiditis (1).

La OMA, en conjunto con la sinusitis aguda y la faringitis por estreptococos del grupo A, corresponden al $50 \%$ de todas las prescripciones de antibiótico de espectro extendido en los niños (6). El sobreuso de antibióticos de amplio espectro además de aumentar el costo económico en el manejo de dichos cuadros clínicos, favorece la resistencia antibiótica (6).

A pesar de que la resistencia a los antibióticos se ha convertido en uno de los problemas de salud pública más acuciantes del mundo, la tasa de prescripción de antibióticos en OMA siguen siendo superior al $90 \%$ (4). Es por lo anterior, que esta revisión tiene como objetivo resumir las pautas actuales de manejo de esta patología y describir los cambios observados en los últimos años en el patrón de resistencia antibiótica de los agentes bacterianos causales de la misma, con el fin de sensibilizar a la comunidad médica sobre la importancia del uso apropiado de antibióticos en OMA.

\section{ETIOLOGÍA}

La causa más frecuente de OMA es viral, siendo los patógenos más comunes el virus respiratorio sincicial, el adenovirus, el rinovirus, entre otros. Dentro de la OMA de causa bacteriana, destacan organismos como: Streptococcus pneumoniae (SP), Haemophilus influenzae no tipificable (Hint), Moraxella catarrhalis (MC) y Streptococcus pyogenes o estreptococos del grupo $A$ (SGA) $(5,7,8)$.

\section{DIAGNÓSTICO $Y$ MANIFESTACIONES CLÍNICAS}

Para el correcto diagnóstico de OMA en niños se requiere de la presencia de (9):

1. Abultamiento moderado-grave de la membrana timpánica u otorrea aguda no debida a otitis externa aguda.

2. Abultamiento leve de la membrana timpánica e inicio reciente (menos de 48 horas) de otalgia o eritema intenso de la membrana timpánica.

3. No se debe diagnosticar OMA en niños que no tienen efusión en el oído medio, basado en la otoscopia neumática y/o la timpanometría.

Los pacientes con OMA a menudo presentan un inicio rápido de fiebre y otalgia, así como signos inespecíficos, entre ellos tirones de orejas, frotamientos, irritabilidad, disminución del sueño y otros cambios de comportamiento. De estos, el dolor suele ser el síntoma más prominente (10). La otalgia como síntoma debe distinguirse del dolor en el canal externo debido a otitis externa (que produce dolor con el movimiento del trago e inflamación del canal auditivo generalmente sin síntomas sistémicos) (11).

Algunos autores refieren que las combinaciones de estas manifestaciones clínicas junto con los hallazgos físicos son clave para diagnosticar la OMA (10). Sin embargo,"la guía de 2013 de la Academia Americana de Pediatría (AAP) sobre diagnóstico y manejo de la OMA" enfatizan la falta de sensibilidad de las escalas de dolor visual 0 las puntuaciones de los síntomas, así como la importancia de la otoscopia para el diagnóstico de OMA. No obstante, la evaluación de los síntomas tienen un 
valor relevante en el seguimiento de la respuesta clínica a la terapia y una adecuada evaluación del dolor no deja de ser importante (12).

\section{MANEJO}

En el año 2013 la AAP emitió recomendaciones sobre el manejo de OMA, según esta publicación el abordaje inicial debe incluir el uso de analgesia en caso de otalgia independientemente de la prescripción de tratamiento antibiótico. Ya que este no provee alivio del dolor durante las primeras 24 horas comparado con placebo e incluso hay pacientes que pueden persistir con dolor 12 días después de iniciado el tratamiento $(9,13)$. Dada la poca evidencia existente, el analgésico a utilizar debe ser seleccionado con base en la relación riesgo-beneficio y tomando en consideración la preferencia del cuidador. En general, se utiliza acetaminofén o ibuprofeno en casos de dolor leve a moderado y narcóticos en dolor moderado a severo. Los agentes tópicos parecen ser eficaces a los 10-30 min de su administración como tratamiento adicional $(9,14)$.

La decisión de iniciar antibioticoterapia de forma inmediata debe fundamentarse en la estratificación de riesgo del paciente según la edad y severidad de los síntomas. Los niños de cualquier edad que presentan síntomas severos (otalgia moderada a severa, otalgia de más 48 horas de evolución o temperatura mayor a $39^{\circ} \mathrm{C}$ ) y los menores de 2 años con afección bilateral, independientemente de la severidad de los síntomas, deben recibir tratamiento antibiótico inmediatamente (9).
Mientras que en aquellos pacientes sin síntomas severos con edades entre 6 y 24 meses y OMA unilateral o mayores de 2 años independiente de la lateralidad, la misma guía brinda una alternativa a la prescripción inmediata de antibióticos denominada espera vigilante. Dicha estrategia consiste en dar seguimiento al paciente por un periodo de 48 a 72 horas antes de iniciar antibioticoterapia dado que cerca del $80 \%$ de los episodios de OMA resuelven espontáneamente dentro de los primeros 3 días $(9,14)$. Es importante destacar que estos criterios aplican para niños sanos con OMA no complicada y en casos donde existe la posibilidad de brindar seguimiento cercano al paciente e iniciar antibioticoterapia ante deterioro clínico o persistencia de los síntomas.

Dichas recomendaciones están basadas en un meta-análisis revisado en el 2015 que evidenció que el uso de antibióticos, comparado con placebo, acelera la resolución de los síntomas, acorta el periodo con efusión del oído medio después de la OMA, disminuye el riesgo de perforación timpánica y de OMA contralateral. Además, los antibióticos parecen ser más efectivos para la resolución de los síntomas en el subgrupo de pacientes con otorrea y en menores de 2 años con afección bilateral. Sin embargo estos beneficios deben ser sopesados con el aumento de riesgo de efectos adversos tales como diarrea, vómitos y rash ampliamente documentado $(13,14,15)$.

Por otro lado, el uso inmediato de antibióticos no se ha asociado con una reducción del dolor a los 3 a 7 días de evolución, anormalidades en la timpanometría a las 4 semanas de evolución, perforaciones de la membrana 
timpánica, recurrencias o complicaciones serias (incluyendo mastoiditis y meningitis) comparado con la estrategia de espera vigilante (13)

En el 2017 se estimó que por cada 1000 pacientes con OMA en los que se implementa la estrategia de espera vigilante de acuerdo con las pautas de la AAP comparado con la práctica actual, se produce una reducción de 514 recetas de antibiótico despachadas de forma inmediata y de 205 ciclos de antibiótico en total, ahorrando así 5573 dólares y evitando 14.3 años de vida ajustados por discapacidad. Está ultima es una medida de la carga de morbilidad expresada en años de vida perdidos por mortalidad prematura 0 cualquier discapacidad causada por una enfermedad (16).

A pesar de que se ha evidenciado que dar instrucciones a los padres de diferir el tratamiento antibiótico efectivamente reduce y retrasa el despacho de antibióticos (en un 39.5\% y $38.9 \%$ respectivamente) y que dicha recomendación fue propuesta por la AAP desde el año 2004, en la práctica actual poco más del $16 \%$ de los padres cuyos hijos califican para espera vigilante reciben instrucciones de aplazar el tratamiento antibiótico (17). Incluso hay estudios que reportan porcentajes menores a $5 \%(16)$.

Pese a que la renuencia de los padres al aplazamiento de la antibioticoterapia pareciera ser uno de los impedimentos identificados para emplear esta recomendación, esta no se ha asociado a un menor grado de satisfacción de los mismos con respecto a la antibioticoterapia inmediata (16).

\section{PRINCIPALES ANTIBIÓTICOS UTILIZADOS EN OMA}

La amoxicilina como tratamiento empírico de la otitis media aguda, sigue siendo el fármaco de primera elección debido a que es altamente efectivo contra las principales cepas de SP, el patógeno predominante en la OMA, así como contra SGA. Es más probable que $\mathrm{MC}$ y algunas cepas de Hint sean resistentes a la amoxicilina (es decir, tienen más probabilidades de producir $\beta$ lactamasas), sin embargo, son patógenos menos comunes (12).

La amoxicilina tiene una excelente penetración en el oído medio (por lo que aún puede ser eficaz a pesar de la resistencia in vitro), es barata, bien tolerada y tiene un espectro antimicrobiano relativamente estrecho. Tiene una vida media corta de aproximadamente 1 hora. Dada en una dosis oral adecuada, la amoxicilina es más efectiva, en comparación con otros antimicrobianos orales contra SP susceptible y resistente a penicilina, $\mathrm{H}$ influenzae $\beta$-lactamasa negativa y SGA (12).

Para la curación clínica de la OMA, los niveles de amoxicilina en el oído medio deben ser adecuados durante más del $50 \%$ del día. La administración de 45-60 $\mathrm{mg} / \mathrm{kg}$ por día de amoxicilina dividida en tres dosis logrará niveles adecuados en el oído medio, mientras que un régimen de dosificación de dos veces por día requiere dosis diarias totales más altas de $75-90 \mathrm{mg} / \mathrm{kg}$ por día para mantener niveles adecuados a lo largo del día (12). La dosis anterior brinda brinda cobertura para el $40-70 \%$ de $H$. influenzae $\beta$ lactamasa negativa y $80-90 \%$ de SP con 
concentraciones inhibitorias mínimas in vitro menores a $4 \mu \mathrm{g} / \mathrm{mL}$ para penicilina (18).

Existen ciertas situaciones clínicas en las que otros antimicrobianos deben considerarse de primera línea. En el contexto de OMA asociada a conjuntivitis purulenta (síndrome de otitisconjuntivitis), Hint y $\mathrm{MC}$ son patógenos comunes; y por lo tanto, el tratamiento combinado de amoxicilina más un inhibidor de $\beta$-lactamasa (por ejemplo, amoxicilina-clavulanato) o una cefalosporina de segunda generación son preferidas (por ejemplo, cefuroxima axetilo) (12).

También puede ser prudente usar amoxicilina-clavulanato si el niño ha recibido amoxicilina en los últimos 30 días, si presenta una probable recaída de una infección reciente o en caso de no respuesta a la amoxicilina (12). Lo anterior porque la amoxicilina-clavulanato proporciona cobertura para más del $98 \%$ de MC (incluidas las cepas productoras de $\beta$-lactamasas), Hint, y conserva la misma eficacia contra SP que la amoxicilina a altas dosis (18).

La ceftriaxona por vía intravenosa o intramuscular se debe reservar para pacientes que no toleran los fármacos orales o en caso de fallo al tratamiento con amoxicilina-clavulanato. En esta última situación, la cual es infrecuente, la ceftriaxona se administra por un período de tres días pues la vida media del fármaco es más larga (aproximadamente de 12 a 24 h); además, debe considerarse el muestreo del líquido del oído medio para cultivo (12).

Si el niño tiene antecedentes de una reacción de hipersensibilidad a la amoxicilina o la penicilina, usar cefalosporinas de segunda generación (cefprozilo o cefuroxima-axetilo) o una cefalosporina de tercera generación es aceptable (cefdinir), a menos que la reacción previa sea mortal (12). El cefdinir posee una actividad algo superior a la amoxicilina para Hint, pero inferior para SP, y se ha estimado una cobertura de aproximadamente $85 \%$ de todos los Hint, la mayoría de MC y $50-75 \%$ de SP con concentraciones inhibitorias mínimas de penicilina menores a $0.5 \mu \mathrm{g} / \mathrm{mL}$. Por otra parte, el costo de cefdinir es hasta 6 veces mayor que el de amoxicilina, pero un $15 \%$ menos que el de amoxicilinaclavulanato (17).

Alternativamente, se puede utilizar un azálido macrólido (claritromicina o azitromicina) o clindamicina. Sin embargo, estos antibióticos generalmente tienen una capacidad bactericida inferior, especialmente para SP y Hint en comparación con los betalactámicos (por ejemplo, penicilinas o cefalosporinas). Solo en raras ocasiones se indican otros medicamentos, como la doxiciclina en niños mayores a 8 años o quinolonas; sin embargo, tales alternativas sólo deben considerarse en consulta con un médico especialista en enfermedades infecciosas (12).

\section{PERFIL DE RESISTENCIA ANTIBIÓTICA}

Los patrones de resistencia antibiótica de las bacterias causantes de OMA varían según la región geográfica, y deben ser la base fundamental sobre la cual se establecen las recomendaciones terapéuticas, motivo por el que es de suma importancia conocer los patrones locales de resistencia antibiótica $(1,2)$. 
El SP resistente a la penicilina se considera en la actualidad la causa principal de fracaso terapéutico de los antibióticos en la OMA (5).

Algunos estudios han evidenciado que en los países donde se aplica la vacuna antineumocócica conjugada heptavalente (PCV7) de forma efectiva y rutinaria, existe una menor proporción de infecciones neumocócicas causadas por los serotipos contenidos en dicha vacuna, y un aumento en la incidencia de otros serotipos como el $19 \mathrm{~A}$ y el 3 $(5,7,19)$.

El estudio realizado en Costa Rica por Abdelnour y colaboradores en 2009, analizó el patrón de resistencia antibiótica del SP en muestras de fluido del oído medio de niños con OM, y se encontró que un $96 \%$ de los aislados de SP serotipo 3 eran susceptibles a penicilina, mientras que únicamente el $79 \%$ de los aislados de otros serotipos eran susceptibles a penicilina. Se observó además, que todos los aislados de SP fueron susceptibles a amoxicilina, cefuroxima, ceftriaxona, faropenem, azitromicina y levofloxacina. Ante estos hallazgos, el grupo investigador indica que a pesar de los cambios en frecuencia de los serotipos de SP, los agentes antibióticos comunes como la amoxicilina, deben seguir considerándose como la primer opción de tratamiento (7).

Se ha establecido que la persistencia del estado portador de este agente en nasofaringe, constituye un factor de riesgo para infecciones recurrentes posteriores, y por tanto, se ha propuesto que el tratamiento antimicrobiano debe asegurar la erradicación de la colonización nasofaríngea (5).
En los últimos 10 años se ha demostrado también, un incremento significativo en la frecuencia de cepas de SP multiresistentes en casos de OMA con fallo del tratamiento, con estudios que muestran incidencias de hasta 84\% (5).

Otro efecto observado con la introducción de la PCV7 es el cambio en la frecuencia de aislamiento entre SP y Hint en pacientes con OMA. En Costa Rica, el aislamiento de SP en pacientes con OM disminuyó de un $42 \%$ entre los años 1999-2001 a un 28\% en el periodo 2002- 2007, mientras que el porcentaje de aislamiento de $\mathrm{H}$. influenzae aumentó de un $14 \%$ a un $23 \%$ (19).

La evidencia actual describe una prevalencia de aislamiento de $H$. influenzae $\beta$-lactamasa positivo en pacientes con OMA, que varía entre menos del $20 \%$ en países como Chile y Venezuela, hasta un $83 \%$ en México. La producción de $\beta$-lactamasas, es el mecanismo responsable de la resistencia bacteriana a antibióticos $\beta$-lactámicos, que constituyen la primera línea de tratamiento para OM, entre los que se encuentran las aminopenicilinas, las cefalosporinas y los carbapenémicos (19).

También se ha reportado el aislamiento de $\mathrm{H}$. influenzae $\beta$-lactamasa negativa resistente a ampicilina (BLNAR, por sus siglas en inglés) en el fluido del oído medio de pacientes con OMA en México, Líbano y Japón. Este mecanismo de resistencia de $\mathrm{H}$. influenzae, corresponde al mecanismo de resistencia a ampicilina más frecuente en el mundo (19).

Con respecto a la $\mathrm{MC}$, este patógeno ha mostrado un aumento significativo en su detección como otopatógeno desde 1980 y la mayor parte de los estudios indican 
que más del $90 \%$ de los aislamientos de MC de pacientes con OMA son productores de $\beta$-lactamasas (19).

El aumento en la incidencia de patógenos productores de $\beta$-lactamasa ha sido la justificación utilizada en los estudios que proponen un abordaje inicial de la OMA con antibióticos de amplio espectro; no obstante, como se mencionó anteriormente, la evidencia ha demostrado que esta práctica no es costo-efectiva, conlleva mayores riesgos asociados, y por lo tanto, las recomendaciones de las guías de manejo de OMA indican que no es conveniente utilizar estos antibióticos como tratamiento empírico (6).

\section{CONCLUSIÓN}

Actualmente, para el diagnóstico de OMA se requiere de síntomas agudos y signos otoscópicos de inflamación del oído medio, más la presencia de efusión en el oído medio documentada mediante otoscopia neumática o timpanometría.

Tras el diagnóstico correcto de OMA el tratamiento con antibioticoterapia de forma inmediata no es siempre justificado. Esto debido a que la espera vigilante es una alternativa segura $y$ costo-efectiva, que puede ser implementada en pacientes con OMA no severa unilateral en menores de 2 años o independientemente de la lateralidad en pacientes mayores. No obstante, en la práctica actual sigue siendo subutilizada. Por otro lado, en los pacientes que ameritan antibioticoterapia, la amoxicilina sigue siendo la primera línea de tratamiento. Esto a pesar de los cambios en la frecuencia de los serotipos de SP inducidos por la PCV7, del incremento de cepas resistentes a $\beta$-lactámicos y por último del aumento de aislamiento de patógenos como Hint y MC.

\section{REFERENCIAS}

1. Abbott P, Gunasekera H, Leach AJ, Askew D, Walsh R, Kong K, Girosi F, Bond C, Morris P, Lujic S, Hu W. A multi-centre open-label randomised non-inferiority trial comparing watchful waiting to antibiotic treatment for acute otitis media without perforation in low-risk urban Aboriginal and Torres Strait Islander children (the WATCH trial): study protocol for a randomised controlled trial. Trials. 2016 Dec; 17(1): 119.https://doi.org/10.1186/s13063-016-1247-y

2. Aguilar-Morales L, Soley-Gutiérrez C, Arguedas-Mohs A. Principios farmacocinéticos y farmacodinámicos en el tratamiento de niños con otitis media. Acta Médica Costarricense. 2006; 48(3): 113-118. Disponible en:http://actamedica.medicos.cr/index.php/Acta Medica/article/view/460/432

3. Park MK, Nam DW, Byun JY, Hong SM, Bae CH, Lee HY, Jeon EJ, Kim MG, Kim SH, Yeo SG. Differences in Antibiotic Resistance of MRSA Infections in Patients with Various Types of Otitis Media. J Int Adv Otol. 2018 Dec 1; 14(3): 459-63. https://doi.org/10.5152/iao.2018.5374

4. Palma S, Rosafio C, Del Giovane C, Patianna VD, Lucaccioni L, Genovese E, Bertolani P, lughetti L. The impact of the Italian guidelines on antibiotic prescription practices for acute otitis media in a paediatric emergency setting. Italian journal of pediatrics. 2015 Dec; 41(1): 37. https://doi.org/10.1186/s13052-015$\underline{0144-4}$

5. Korona-Glowniak I, Zychowski P, Siwiec R, Mazur E, Niedzielska G, Malm A. Resistant Streptococcus pneumoniae strains in children with acute otitis media-high risk of persistent colonization after treatment. BMC infectious diseases. 2018 Dec; 18(1): 478. https://doi.org/10.1186/s12879-018-3398-9 
6. Gerber JS, Ross RK, Bryan M, Localio AR, Szymczak JE, Wasserman R, Barkman D, Odeniyi F, Conaboy K, Bell L, Zaoutis TE. Association of broad-vs narrow-spectrum antibiotics with treatment failure, adverse events, and quality of life in children with acute respiratory tract infections. Jama. 2017 Dec 19; 318(23): 2325-2336. https://doi.org/10.1001/jama.2017.18715

7. Abdelnour A, Soley C, Guevara S, Porat N, Dagan R, Arguedas A. Streptococcus pneumoniae serotype 3 among Costa Rican children with otitis media: clinical, epidemiological characteristics and antimicrobial resistance patterns. BMC pediatrics. 2009 Dec; 9(1): 52. https://doi.org/10.1186/1471-2431-9-52

8. Krause FJ. Otitis media aguda. Diagnóstico y manejo práctico. Revista Médica Clínica Las Condes. 2016 Nov 1; 27(6): 915-923. https://doi.org/10.1016/j.rmclc.2016.10.004

9. Lieberthal AS, Carroll AE, Chonmaitree T, Ganiats TG, Hoberman A, Jackson MA, Joffe MD, Miller DT, Rosenfeld RM, Sevilla XD, Schwartz RH. The diagnosis and management of acute otitis media. Pediatrics. 2013 Mar 1; 131(3): e964-e999. https://doi.org/10.1542/peds.2012-3488

10. Rosa-Olivares J, Porro A, Rodriguez-Varela M, Riefkohl G, Niroomand-Rad I. Otitis Media: To Treat, To Refer, To Do Nothing: A Review for the Practitioner. Pediatrics in review. 2015 Nov; 36(11): 480486.https://doi.org/10.1542/pir.36-11-480

11. Le Saux N, Robinson JL, Canadian Paediatric Society, Infectious Diseases and Immunization Committee. Management of acute otitis media in children six months of age and older. Paediatrics \& child health. 2016 Feb 5; 21(1): 39-44. https://doi.org/10.1093/pch/21.1.39

12. Kirk LA. Pediatric Otitis Media: An Update. Physician Assistant Clinics. 2018 Apr 1; 3(2):207222. https://doi.org/10.1016/i.cpha.2017.11.004

13. Venekamp RP, Sanders SL, Glasziou PP, Del Mar CB, Rovers MM. Antibiotics for acute otitis media in children. Cochrane Database of Systematic Reviews. 2015; (6): CD000219.https://doi.org/10.1002/14651858.CD000219.pub4

14. Venekamp RP, Damoiseaux RA, Schilder AG. Acute otitis media in children. BMJ clinical evidence. 2014 Sep; 2014: pii 0301.

15. Schilder AG, Marom T, Bhutta MF, Casselbrant ML, Coates H, Gisselsson-Solén M, Hall AJ, Marchisio P, Ruohola A, Venekamp RP, Mandel EM. Panel 7: otitis media: treatment and complications. Otolaryngology-Head and Neck Surgery. 2017 Apr; 156(4_suppl): S88S105. https://doi.org/10.1177/0194599816633697.

16. Sun D, McCarthy TJ, Liberman DB. Cost-effectiveness of watchful waiting in acute otitis media. Pediatrics. 2017 Apr 1; 139(4): e20163086. https://doi.org/10.1542/peds.2016-3086

17. Brinker Jr DL, MacGeorge EL, Hackman N. Diagnostic Accuracy, Prescription Behavior, and Watchful Waiting Efficacy for Pediatric Acute Otitis Media. Clinical pediatrics. 2019 Jan; 58(1): 6065.https://doi.org/10.1177/0009922818806312

18. Harrison CJ. Amoxicillin is the most cost-effective therapy for acute otitis media: the culmination of 40 years of research. The Journal of pediatrics. 2017 Oct 1; 189: 58. https://doi.org/10.1016/.jpeds.2017.06.070

19. Ngo CC, Massa HM, Thornton RB, Cripps AW. Predominant Bacteria Detected from the Middle Ear Fluid of Children Experiencing Otitis Media: A Systematic Review. PLoS One. 2016; 11(3): e0150949.https://doi.org/10.1371/journal.pone.0150949 